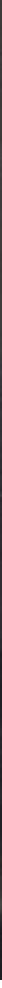

Szene aus Geigers Inszenierung der Oper „Aida" von Giuseppe

Verdi, die am 9. September 1956 Premiere am Großen Haus Dresden feierte

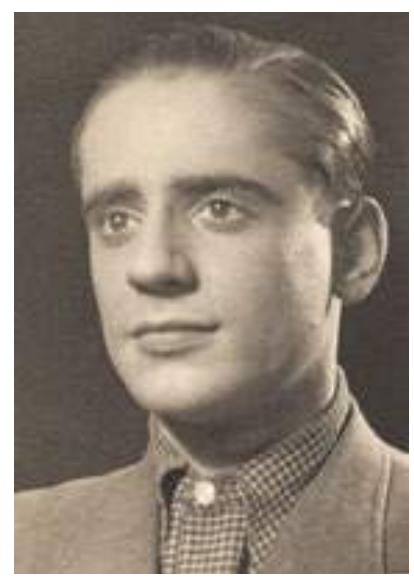

Der junge Erich Geiger 1946 in Karlsruhe

\title{
Der Dresdner Nachkriegsregisseur Erich Geiger
}

\author{
Romy Petrick
}

Der Regisseur und Bühnenautor Erich Geiger ist heute weitgehend unbekannt. Geboren 1924 in Karlsruhe, wuchs er während der Zeit der Weimarer Republik und des Nationalsozialismus auf und begann unmittelbar nach Kriegsende mit einer fulminanten Karriere als Regisseur. Von 1955 bis 1965 wirkte er als Oberspielleiter an der Dresdner Staatsoper. 1965 verließ er die DDR. Die Besonderheit seines Schaffens liegt in der Tatsache, dass er im Ost- wie im Westteil Deutschlands gleichermaßen arbeitete und letztendlich in dem politischen Spannungsfeld scheiterte. Sein vielseitiges Wirken umfasste alle Genres (Opern, Operetten, Schauspiel, Musical). Außerdem arbeitete er bei den neu entstehenden Fernsehsendern in Ost- und Westdeutschland. Dazu schrieb er Drehbücher, Schauspiele, Libretti und später auch Ratgeber zu verschiedenen Themen. Von 1995 bis 2005 leitete er die Dresdner Seniorenakademie und wurde im Jahr 2000 mit dem Verdienstorden des Freistaates Sachsen ausgezeichnet. 2015 erschien seine umfassende Biographie.

\section{Kindheit und Jugend}

Erich Geiger entstammte einem gutsituierten Elternhaus. Er war das einzige Kind des Mediziner-Ehepaares Regina Renate Geiger (18941965) und Alois Michael Geiger (1893-1965). Bereits während der Schulzeit hatte Erich Geiger ab 1940 Kurse an der Karlsruher Musikhochschule in Musiktheorie und Dirigieren absolviert und folgte nach dem Abitur 1942 seinen eigenen Ambitionen: Er begann gegen den Willen seines Vaters am 1. Oktober 1942 ein Volontariat als Korrepetitor und Kapellmeister am Badischen Staatstheater Karlsruhe. Dort half er auch als Regieassistent aus und lernte im Alter von 19 Jahren seine erste Frau - die 14 Jahre ältere Opernsängerin Margarethe Lindner (geb. 1910) - kennen. Nach Kriegsende begann Geiger ein Medizinstudium in Heidelberg und übernahm zusätzlich eine Stelle als Regieassistent bei Gustav Hartung (1887-1946), wo er bis zu dessen Tod im Februar 1946 arbeitete. Hartung war einer der wichtigsten Vertreter des expressionistischen Theaters und prägte Geiger in dieser kurzen gemeinsamen Zeit enorm. Besonders der Um- 
gang mit Beleuchtungseffekten (Licht und Schatten) wurde von Erich Geiger übernommen und zum elementaren Stilmittel seiner Inszenierungsarbeit.

1946 kehrte Geiger nach Hartungs Tod an das Badische Staatstheater zurück, wo er als Schauspieler und Regieassistent aushalf. Große Aufmerksamkeit erregte er in einer Hauptrolle als „Rolf Mamlock“ in dem Stück „Professor Mamlock“ von Friedrich Wolf (1888-1953). Der Autor, der bei der Premiere zugegen war, empfahl Geiger daraufhin nach Berlin.

\section{Berliner Jahre (1946-1953)}

Im August 1946 übernahm Geiger die Position des Chefdramaturgen und Spielleiters unter der Leitung des Intendanten Fritz Wisten (1890-1962) am „Theater am Schiffbauerdamm“, wo er bis Juli 1949 tätig war. Wisten leitete hier die Volksbühne, da die Berliner Volksbühne am Rosa-Luxemburg-Platz zerstört worden war. Geiger wählte Stücke aus, schrieb Artikel, gestaltete Programmhefte, bearbeitete mehrere Werke und inszenierte auch drei Produktionen. Die Zeitschrift „Der Start" nannte den Dramaturgen Geiger einen „theaterbesessenen jungen Menschen“, der mit „energischem Elan“ die „müde Bequemlichkeit“ der Theater mit „avantgardistischen Aufführungen“ aufbrechen wolle. ${ }^{1}$ Auf Geigers Initiative hin wurde die Matineen-Reihe „Drama der Zeit“ ins Leben gerufen, die monatlich jeweils Sonntagvormittag um 11 Uhr stattfinden sollte. Geiger sammelte talentierte junge Schauspieler um sich, die ohne Honorar spielten. Zu ihnen zählte u. a. auch der 19-jährige Harry Wüstenhagen (1928-1999), der später Karriere machte, und der erst zwanzigjährige Martin Benrath (1926-2000). Geiger entdeckte dadurch viele Schauspieler der Nachkriegszeit.

Geiger begann zusätzlich an anderen Theatern als Gastregisseur zu inszenieren. Dazu zählte das Mecklenburgische Staatstheater Schwerin, wo Geiger in der Spielzeit 1948/49 vier Stücke herausbrachte. Im Herbst 1949 inszenierte er erstmals auch in Dresden: Für den Ortsverein der Volksbühne brachte er die von ihm bearbeitete Stegreifkomödie „Die schlaue Susanne“ des spanischen Dichters Lope de Vega (1562-1635) heraus. Geiger verzichtete weitestgehend auf ein Bühnenbild und arbeitete mit nur einigen beweglichen Versatzstücken. Die Schauspieler traten im Straßenanzug auf und wechselten auf der Szene die Kostüme. Dies war ein typisches Gestaltungsmittel Geigers, das er bei vielen späteren Inszenierungen anwandte. Im Oktober 1949 gründete Geiger sein eigenes Theater in West-Berlin: Das von der amerikanischen Besatzungsmacht mit 2.000,DM bezuschusste „Experimentiertheater“.
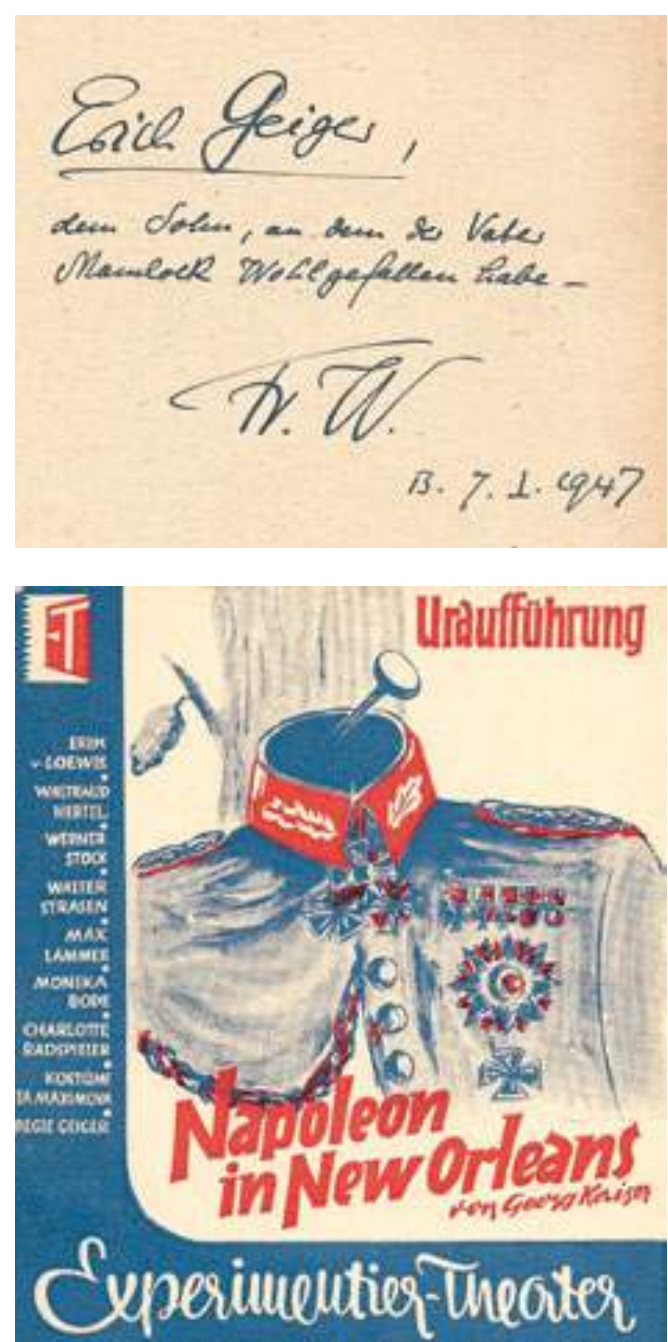

IM HAUS AM WALDSEE * BERIIN-ZEHIENDORF

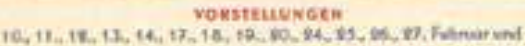

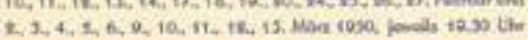

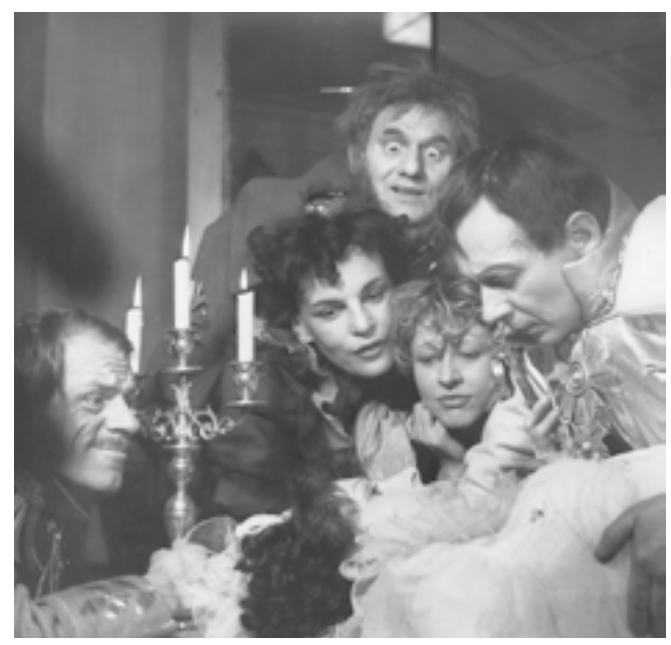

Widmung des Schriftstellers Friedrich Wolf in einer Ausgabe seines Buches „Professor Mamlock", 1947

Plakat zur Uraufführung des Stückes "Napoleon in New Orleans" am Experimentiertheater in West-Berlin, 1950

Die Hauptdarsteller von "Napoleon in New Orleans" am Experimentiertheater, Berlin 1950 
Schon der Name war Programm: Hier sollten Theater-Experimente stattfinden, die sich von der Tradition verabschiedeten und sowohl inhaltlich als auch inszenatorisch neue Wege beschreiten würden. Geiger wollte sich vor allem vom Prinzip der „Guckkastenbühne" lösen und den Theater- mit dem Zuschauerraum verschmelzen. Insgesamt wurden drei Stücke dort zur Aufführung gebracht, die ein enormes mediales Echo hervorriefen. Aus finanziellen Gründen musste das Theater jedoch bereits wenige Monate nach Eröffnung wieder schließen.

Hans Pitra (1915-1977), der neue Intendant des „Metropoltheater“ war jedoch auf den jungen Regisseur und sein Experimentiertheater aufmerksam geworden und verpflichtete ihn als Spielleiter. Seine erste Inszenierung an diesem Theater war die Operette „Die Banditen“ von Jacques Offenbach (1819-1880). Geiger versuchte trotz der im Osten herrschenden Zensur etwas Außergewöhnliches zu schaffen und Kritik an den alltäglichen Problemen in der DDR zu üben. Die Ost- und West-Presse reagierte darauf sehr unterschiedlich. Alle Rezensenten waren sich aber darin einig, dass diese Art von Operetten-Inszenierung etwas völlig Neuartiges sei. So wurde auch der bekannte Regisseur Walter Felsenstein (19011975) auf Geiger aufmerksam, der ihn im August 1951 als Chefdramaturg an die Komischen Oper Berlin verpflichtete. Es folgten nun Einladungen an größere Theater, u. a. an die Oper Leipzig, wo Geiger unter der musikalischen Leitung von Franz Konwitschny (1901-1962)

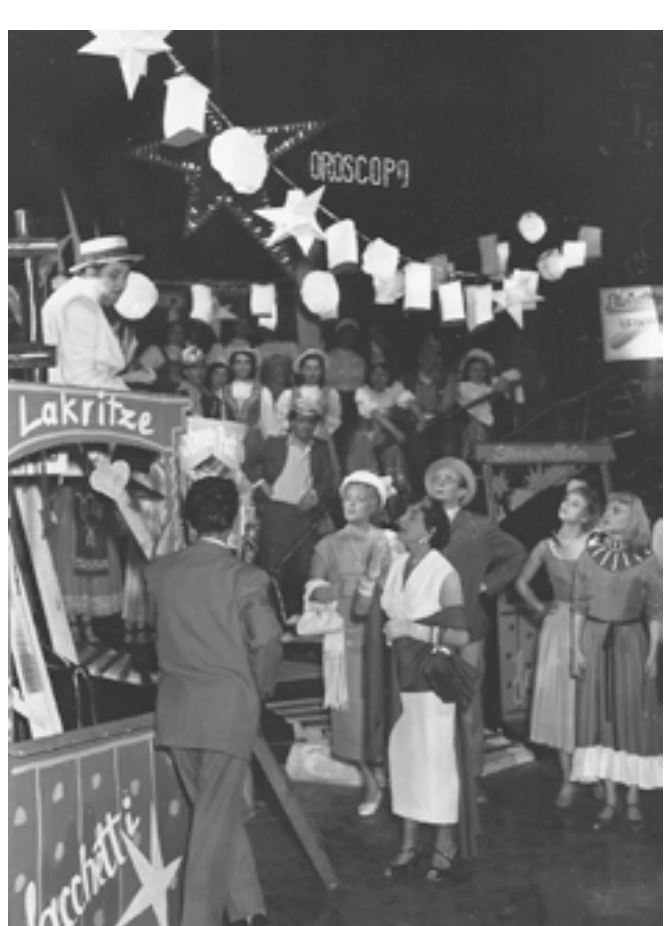

Richard Strauss ` „Rosenkavalier“ und Puccinis „La Bohème“ inszenierte oder an das Opernhaus Halle, wo er die Uraufführung „Jahrmarkt der Illusionen“ des polnischen Autors Julian Tenard herausbrachte.

Geiger konnte direkt an der Arbeit des schon damals bekannten Regisseurs Walter Felsenstein partizipieren. Im Dezember 1952 erhielt Geiger sogar die Gelegenheit, an der Komischen Oper Berlin Puccinis „La Bohème“ textlich $\mathrm{zu}$ bearbeiten und selbst neu $\mathrm{zu}$ inszenieren. Seine Berliner Inszenierung beeindruckte die Kritik, doch muss sich die Probenarbeit sehr schwierig gestaltet haben. Felsenstein wandte sich daraufhin von Geiger ab und man beendete im Januar 1953 die Zusammenarbeit. Enttäuscht verließ Geiger die DDR und nahm eine Stelle als Oberspielleiter an den neugeründeten Vereinigten Bühnen Krefeld/Mönchengladbach an, wo er in der Spielzeit 1953/54 sieben Opern-Inszenierungen herausbrachte. Nebenbei begann er als Dramaturg beim Fernsehen des Nordwestdeutschen Rundfunks (NWDR) zu arbeiten und erhoffte sich dort eine Anstellung. Aufgrund einer Inszenierung an der Staatoperette Dresden beendete der NWDR jedoch die Zusammenarbeit mit Geiger, da man ihm politische „Zweigleisigkeit“ vorwarf.

\section{Dresdner Jahre 1954 bis 1965}

In Dresden hatte Geiger mit „Der Graf von Luxemburg“ am Operettentheater Leuben einen großen Erfolg gefeiert und erhielt nun

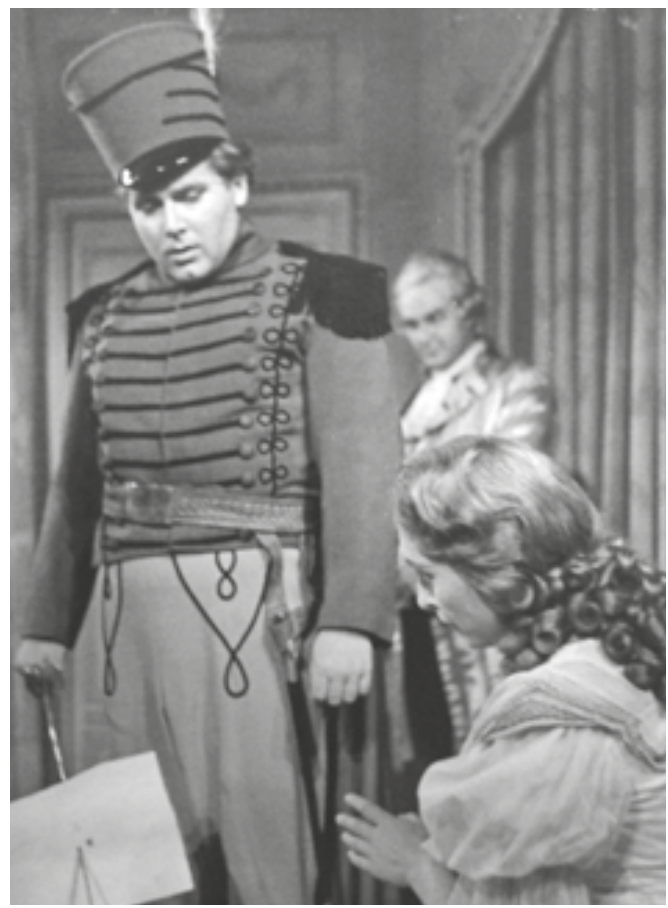

LInks: Szene aus Geigers Operette "Sterne, Gold und Vagabunden" mit einer Musik von Herbert Kawan, Dresden 1956

Rechts: Peter Schreier in Geigers Inszenierung von Mozarts "Cosi fan tutte", Dresden 1963 


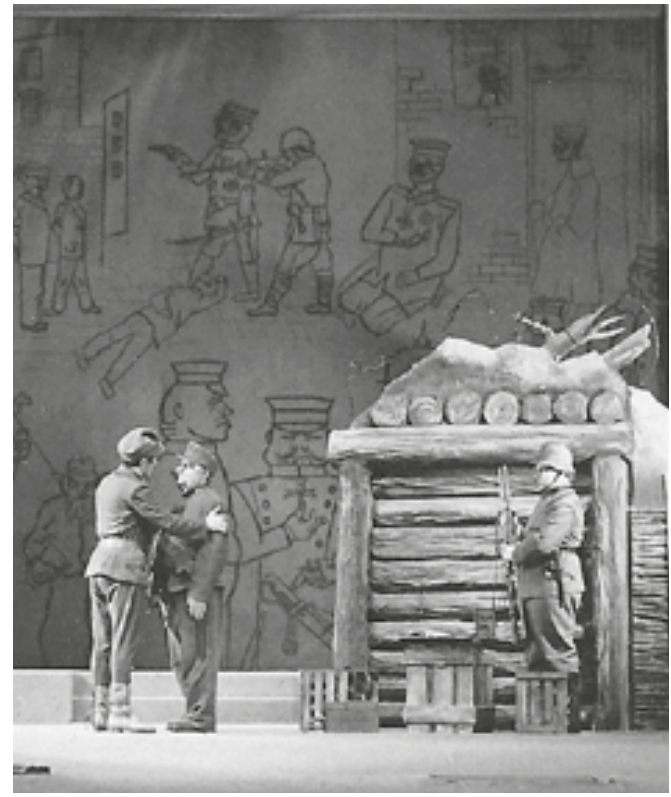

weitere Aufträge. So schrieb er für die Staatsoper die frühe Mozart-Oper „Lucius sulla“ um und brachte am Operettentheater eine eigene Operette unter dem Titel „Sterne, Geld und Vagabunden“ mit einer Musik von Herbert Kawan (1903-1969) heraus.

Der damalige Intendant der Staatsoper Dresden, Dr. Heinrich Allmeroth (1901-1961), verpflichtete Geiger 1954 als Oberspielleiter an das traditionsreiche Theater, dessen Gebäude 1945 zerstört worden war. Der Spielbetrieb wurde bis 1985 im Großen Haus des Staatsschauspiels durchgeführt. Geiger blieb bis 1965 in dieser Funktion und inszenierte in dieser Zeit allein am Großen Haus 17 Opern. Im Kleinen Haus auf der Glacisstraße brachte er mindestens sechs Opern auf die Bühne, wobei er auch viele Schauspiele inszenierte.

Teilweise wurden seine Schauspielinszenierungen vom Deutschen Fernsehfunk übertragen und machten das Dresdner Schauspie-

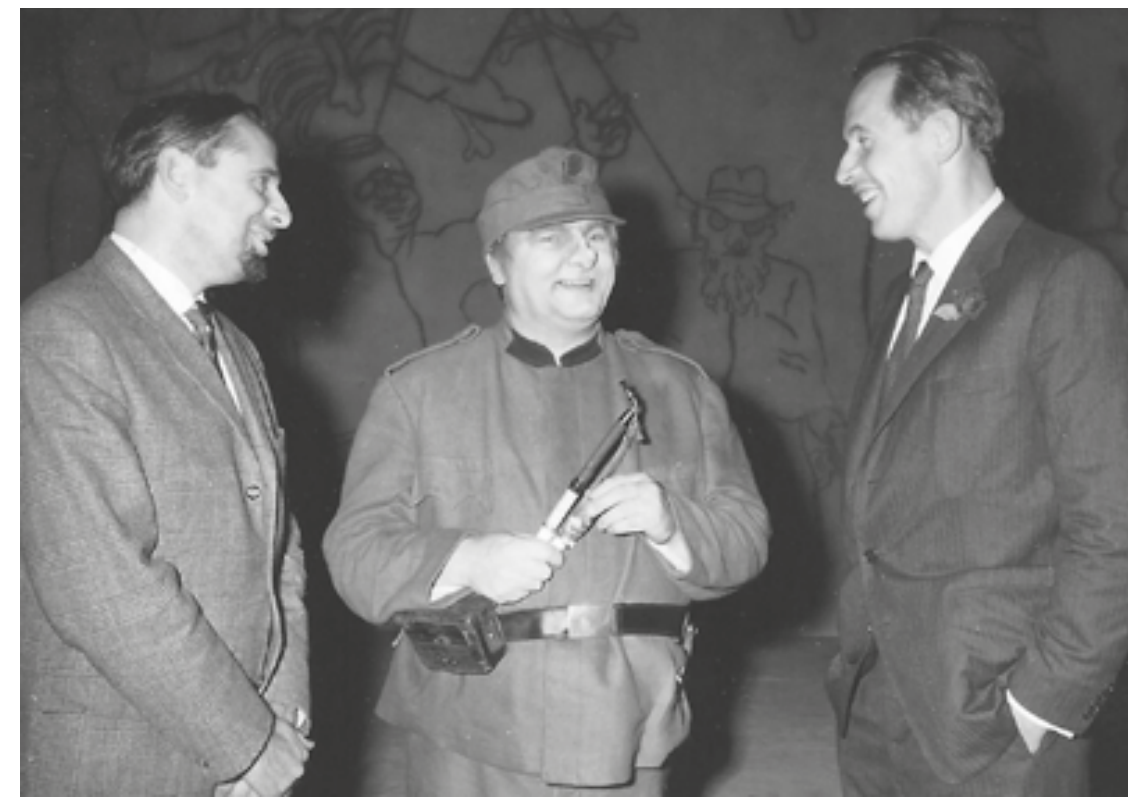

lensemble DDR-weit bekannt. Geiger begleitete dabei die Karriere von vielen jungen Sängern und Schausielern der DDR, zu denen so berühmte Künstler wie Theo Adam (geb. 1926) oder Peter Schreier (geb. 1935) gehören.

$\mathrm{Zu}$ seinen wichtigsten Inszenierungen in Dresden zählen die Uraufführung von KarlRudi Griesbachs musikdramatischem Werk „Der Schwarze, der Weiße und die Frau“ (8. Dezember 1963), die Erstaufführung vom Leos Janáčeks (1854-1928) Oper „Aus einem Totenhaus“ (29. Januar 1960) und die Erstaufführung von Robert Kurkas (19211957) „Der brave Soldat Schwejk“ (10. November 1959).

Mit diesem Werk unternahm die Dresdner Staatsoper ihr erstes Gastspiel in die Bundesrepublik Deutschland. Der Unternehmer Philipp Rosenthal (1916-2001) hatte das renommierte Ensemble zu einem sogenannten „Rosenthaler Feierabend“ eingeladen,
Links: Szene aus Geigers Inszenierung "Der brave Soldat Schweijk" von Robert Kurka am Kleinen Haus Dresden, 1962

Rechts: Erich Geiger, Hauptdarsteller Karl-Heinz Thomann und Philipp Rosenthal in Selb

Unten: Das Große Haus und das Kleine Haus in Dresden, Wirkungsstätten Erich Geigers von 1955-1965, 1953 Fotos: SLUB Dresden, Deutsche Fotothek
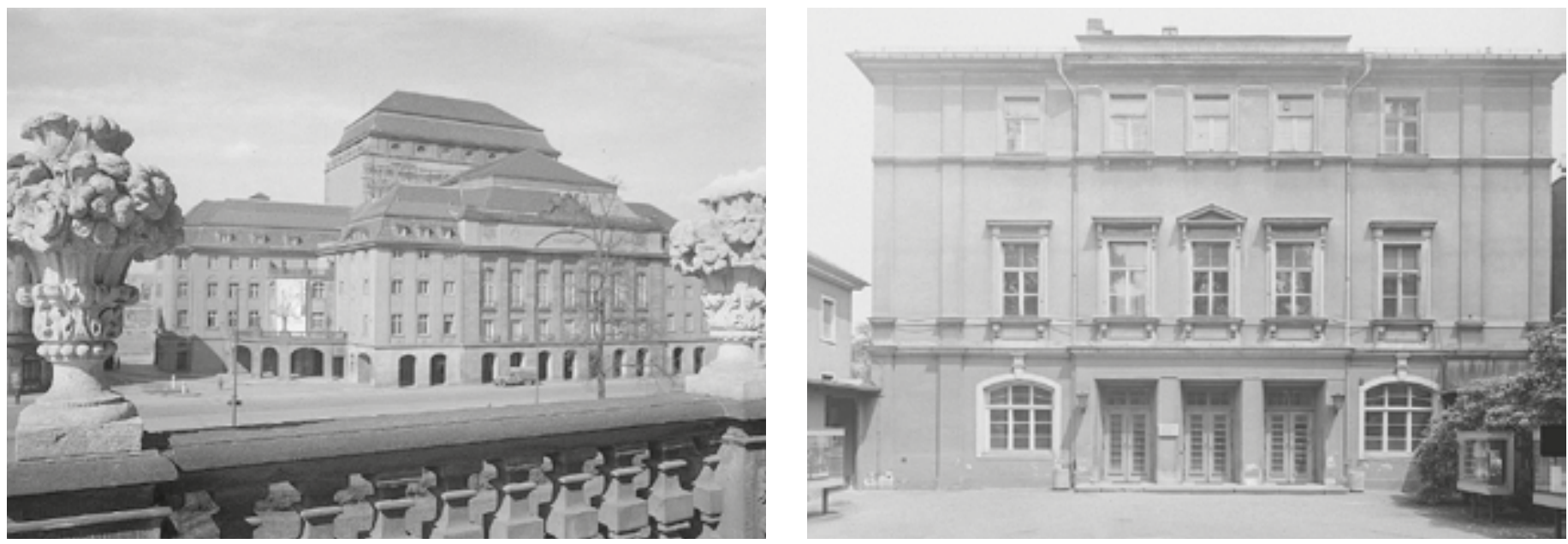
Titelbild des Rundfunk- und Fernsehprogramms des DFF mit Ruth Maria Kubitschek als „Carmen“, 1957

Aufenthaltsgenehmigungen für den Bürger der Bundesrepublik Deutschland, Erich Geiger, in der DDR, 1957 der regelmäßig für die Mitarbeiter und Interessierte veranstaltet wurde. Viele internationale Künstler wie Maria Callas (19231977), Louis Armstrong (1901-1971) oder Yehudi Menuhin (1916-1999) waren dort zu Gast. Dieses Gastspiel wurde für die Staatsoper und für Geiger zu einem großen Erfolg. Das Ensemble der Staatsoper Dresden hatte somit die deutsche oder gar europäische Erstaufführung von Kurkas Oper in Ost- und Westdeutschland übernehmen können. Der junge Regisseur wurde daraufhin weiter nach Selb verpflichtet und gründete dort eine Betriebs-Laienschauspielgruppe, mit der Aufführungen geplant wurden. Da im August 1961 allerdings die Berliner Mauer errichtet wurde, konnte Geiger die Probenarbeit in Selb nicht fortführen und musste in der DDR verbleiben. Zudem verstarb am 18. Oktober 1961 Generalintendant Dr. Heinrich Allmeroth und Geiger verlor mit ihm einen wichtigen Fürsprecher. Stattdessen wurde im Februar 1962 Gerd Michael Henneberg (19222011) mit der Generalintendanz der Staatstheater Dresden betraut, der die Arbeitsweise Geigers stark kritisierte. Es kam zu mehreren sogenannten Kader-Gesprächen, in welchen sich Geiger für seine Arbeitsweise rechtfertigen musste. Da er kaum politisch aktiv war, sondern im Gegenteil seine Kontakte nach Westdeutschland immer aufrechterhalten hatte, wurde er zum unbequemen Außenseiter. 1964 verlängerte man deshalb seinen Vertrag an der Staatsoper Dresden nicht.

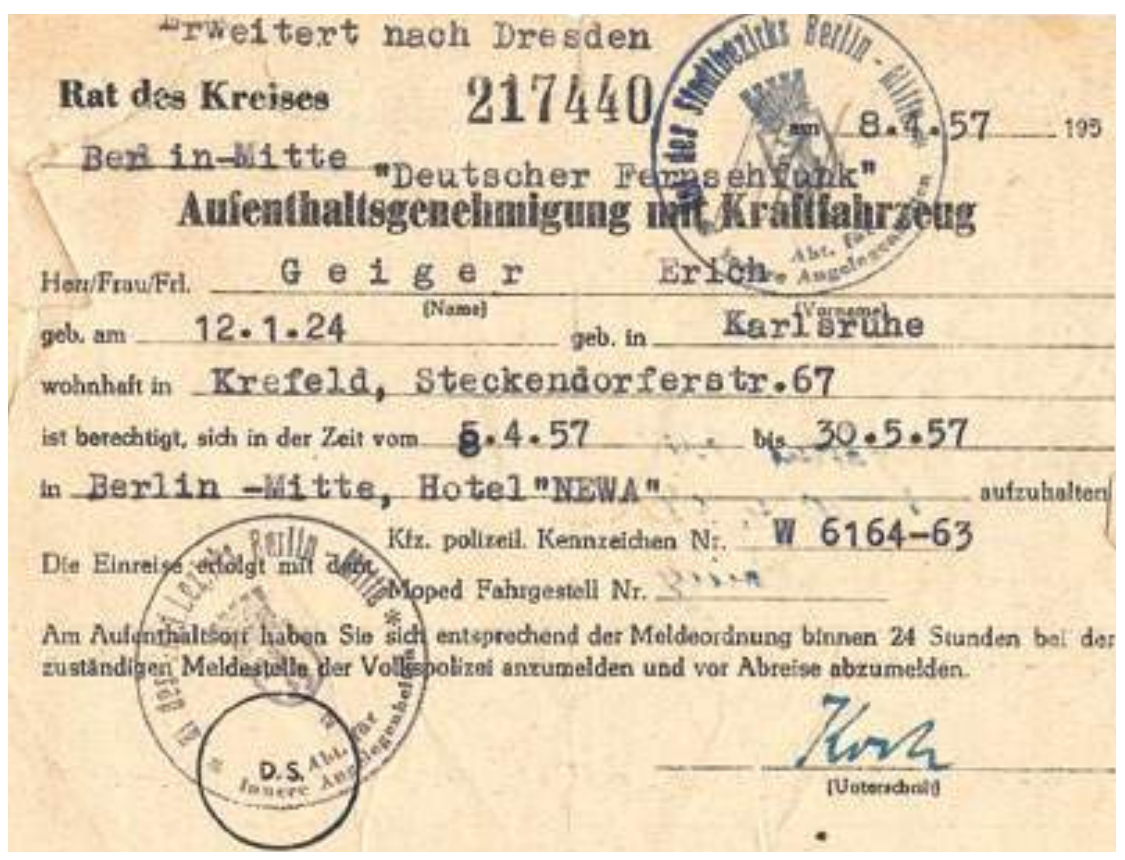

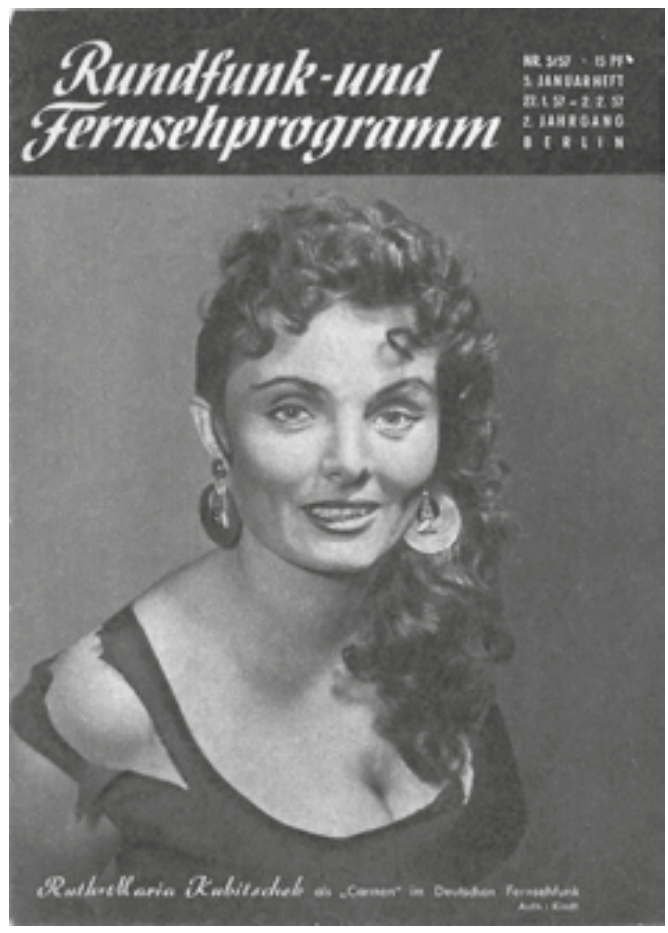

Fernsehopern in den 1950er Jahren

Bereits Anfang der 1950er Jahre hatte Geiger sich intensiv mit dem Medium Fernsehen auseinandergesetzt. Nach der gescheiterten Zusammenarbeit mit dem NWDR in Westdeutschland, begann Geiger 1950 für den DFF in der DDR zu arbeiten. Für ihn war vor allem die filmische Umsetzung von Musik, insbesondere von Opern, zur Aufgabe geworden. So inszenierte er die ersten Fernsehopern, die in der DDR ausgestrahlt wurden. Dazu gehörten u. a. „La Bohéme“ (1955), „Carmen“ (1956), „Tosca“ (1959) und „Oberon“ (1962). Erich Geiger bekam für diese Projekte die neueste Technik und ein großes Studio in Berlin-Adlerhorst zur Verfügung gestellt. Diese ersten DDR-Opernfilme wurden live gesendet und darstellerisch von Schauspielern gestaltet, die sich zur Musik bewegten und spielten. Die Schauspieler sangen auswendig die gesamte Partie mit, um so glaubwürdig die Rolle eines Sängers zu verkörpern. Eine lange Probenphase ging diesen Sendungen voraus, und es erfolgte eine Direktübertragung. Der große logistische Aufwand einer derartigen Produktion ist kaum nachvollziehbar, da alle Aktionen sekundengenau geprobt und umgesetzt werden mussten. Für „Carmen“ engagierte Geiger die junge Schauspielerin Ruth Maria Kubitschek (geb. 1931), die kurz darauf die DDR verließ und in der Bundesrepublik Deutschland eine große Karriere begann. 


\section{Ausreise und Neubeginn}

Nachdem Geigers Vertrag an der Staatsoper Dresden am 31. Juli 1965 ausgelaufen war und er aufgrund eines Autounfalls seiner Eltern im Mai 1965 im Sommer 1965 die DDR verlassen hatte, schien für ihn eine Wiedereinreise in die DDR wenig sinnvoll. Geiger, der selbst aufgrund seiner Epilepsieerkrankung eine umfassende medizinische Betreuung benötigte, wusste, dass er nach einer Rückkehr in die DDR diese nicht wieder würde verlassen können und entschied sich deshalb bewusst dafür, in Westdeutschland zu bleiben. In der Bundesrepublik Deutschland stellte er diese Entscheidung jedoch anders dar und verbreitete stattdessen die Auffassung, dass man ihm eine Wiedereinreise in die DDR verweigert hätte. Um seine zweite Ehefrau Carola und den gemeinsamen Sohn in die Bundesrepublik Deutschland zu holen, drohte Geiger mit der Veröffentlichung von mehreren Artikeln über Suizide unter Künstlern in der DDR und verlangte für die Unterlassung dieser Veröffentlichungen die Ausreisegenehmigung für seine Familie. Weihnachten 1965 wurde seiner Frau und seinem Sohn daraufhin die Ausreise nach West-Berlin gestattet.

In der Bundesrepublik musste sich Geiger nun völlig neu orientieren. Er versuchte an alte Kontakte anzuknüpfen und begann an der Kleinen Oper Berlin zu arbeiten, wo er seine Version von „Lucius sulla“ 1967 nochmals herausbrachte.

Des Weiteren arbeitete er am Theater Ulm. Er wandte sich auch wieder an das Theater Krefeld/Mönchengladbach, wo er mehrere Stücke inszenierte. In der Spielzeit 1967/ 1968 übernahm er sogar die Position des Oberspielleiters an den Städtischen Bühnen Münster, wo er die deutsche Erstaufführung von Benjamin Brittens Oper „Gloriana“ mit der Sängerin Martha Mödl (1912-2011) in der Hauptrolle inszenierte. Anschließend erhielt er sogar eine Einladung an das damals renommierte Theater Bremen, wo er zwei Musicals inszenieren konnte. Allerdings kam es dort zu Konflikten, und er beendete die $\mathrm{Zu}-$ sammenarbeit vorzeitig. Stattdessen wandte er sich wieder dem Medium Fernsehen zu und begann 1970 als Dramaturg bei der Ullstein AV zu arbeiten, wo Sendungen für das Fernsehen produziert wurden. 1973 musste diese Firma allerdings Insolvenz anmelden. Für Geiger begann nun eine finanziell sehr unsichere Zeit, in der er durch Immobilienspekulationen viel Geld verlor. Auch im privaten Bereich nahmen die Probleme überhand, so-

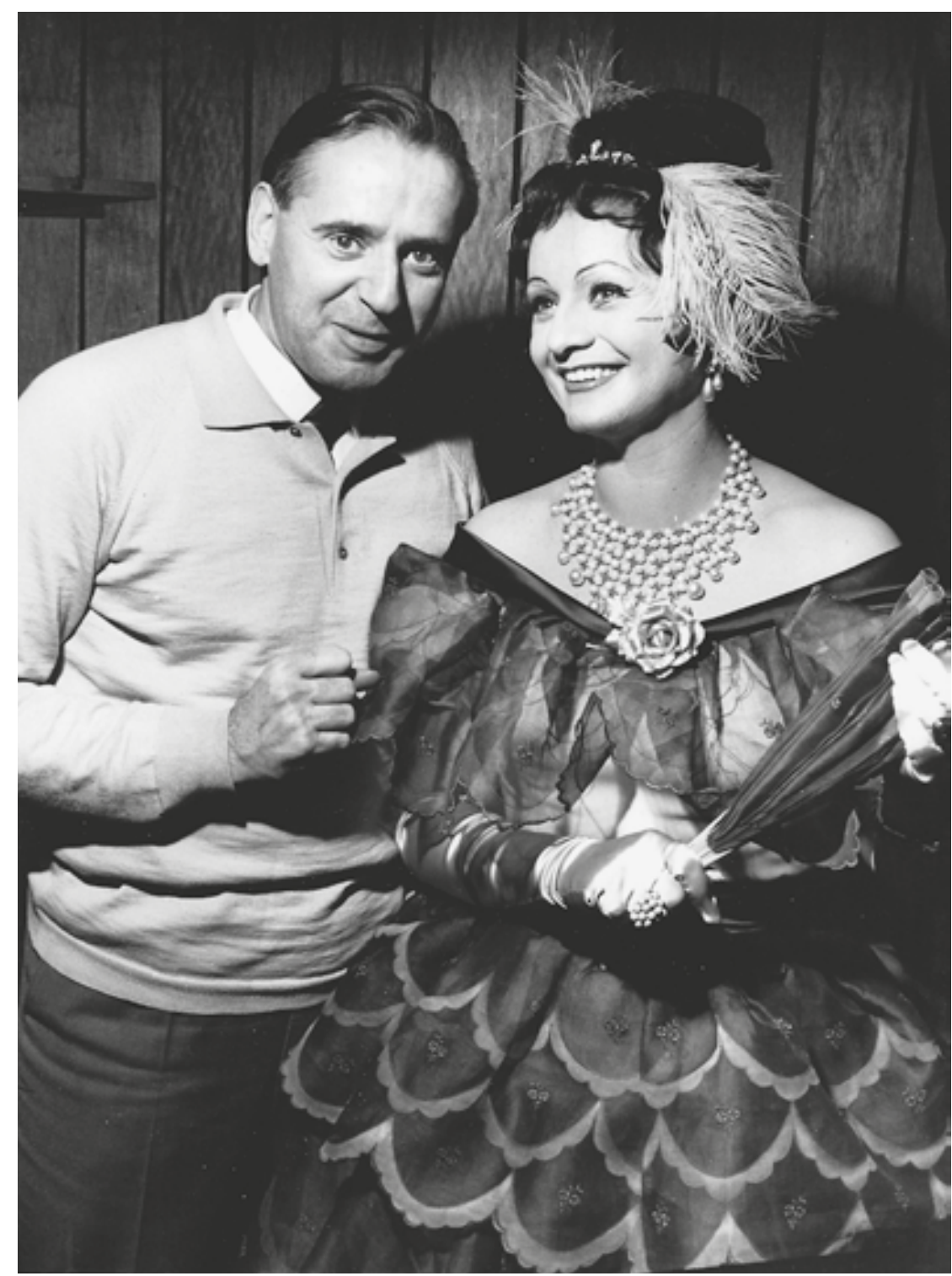

dass er in seiner vermeintlich ausweglosen Situation 1975 einen Selbstmordversuch unternahm. Danach änderte sich sein Leben grundlegend: er wandte sich vom Theaterund Fernsehmetier ab und begann einen neuen Lebensabschnitt mit seiner dritten Ehefrau Edelgard, geb. Kummerfeldt (1935-2014). Gemeinsam gründeten sie eine Hundepension bei Hamburg und Geiger veröffentlichte mehrere Bücher zum Thema Tierhaltung. ${ }^{2}$

\section{Rückkehr nach Dresden}

Im November 1989 unternahm Erich Geiger mit seiner Frau eine Reise nach Dresden und konnte so hautnah die politischen Veränderungen miterleben. Nach der Wiedervereinigung bemühte er sich um die Rückübertragung seiner Villa in Cossebaude, in die er 1994 mit seiner Familie zog. Geiger brachte sich in den 1990er Jahren intensiv
Erich Geiger mit der Hauptdarstellerin der Oper "Don Pasquale" an der Kleinen Oper Berlin, 1966
2 Von Erich Geiger sind z. B. erschienen: Lord York mit Schleife. Heitere Yorkshire Geschichten. Von einer Land- schaft und ihren Hunden. Hamburg 1979, Spaß in Weiß. Heiteres vom Tennis. Ham- burg 1980, Das ist zum Wie- hern. Mit Pferde-Tierzeichen als wären sie Menschen. Ham- burg 1981, Sonderangebot Traumhaus. Heiteres über die Tücken des Objekts beim Hauskauf. Hamburg 1982, Das ist Spitze. Ein Bilder-Quiz zum Mitmachen. Hamburg 1983, Tiere in Pension. Ham- burg 1990.


Prof. Hermann Kokenge, Erich Geiger, Prof. Dr. Achim Mehlhorn und Hannes Lehmann zum 80. Geburtstag von Erich Geiger Foto: Prof. Dr. Achim Mehlhorn, 2004

3 Prof. Dr. Achim Mehlhorn im Gespräch über Erich Geiger, 16. September 2015

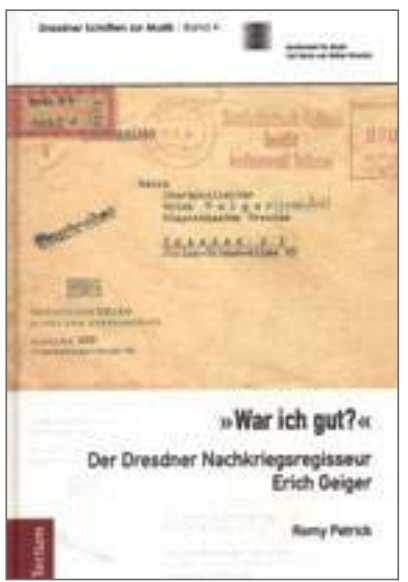

Romy Petrick: „War ich gut?". Der Dresdner Nachkriegsregisseur Erich Geiger, Marburg 2015, 220 Seiten, 29,95 Euro

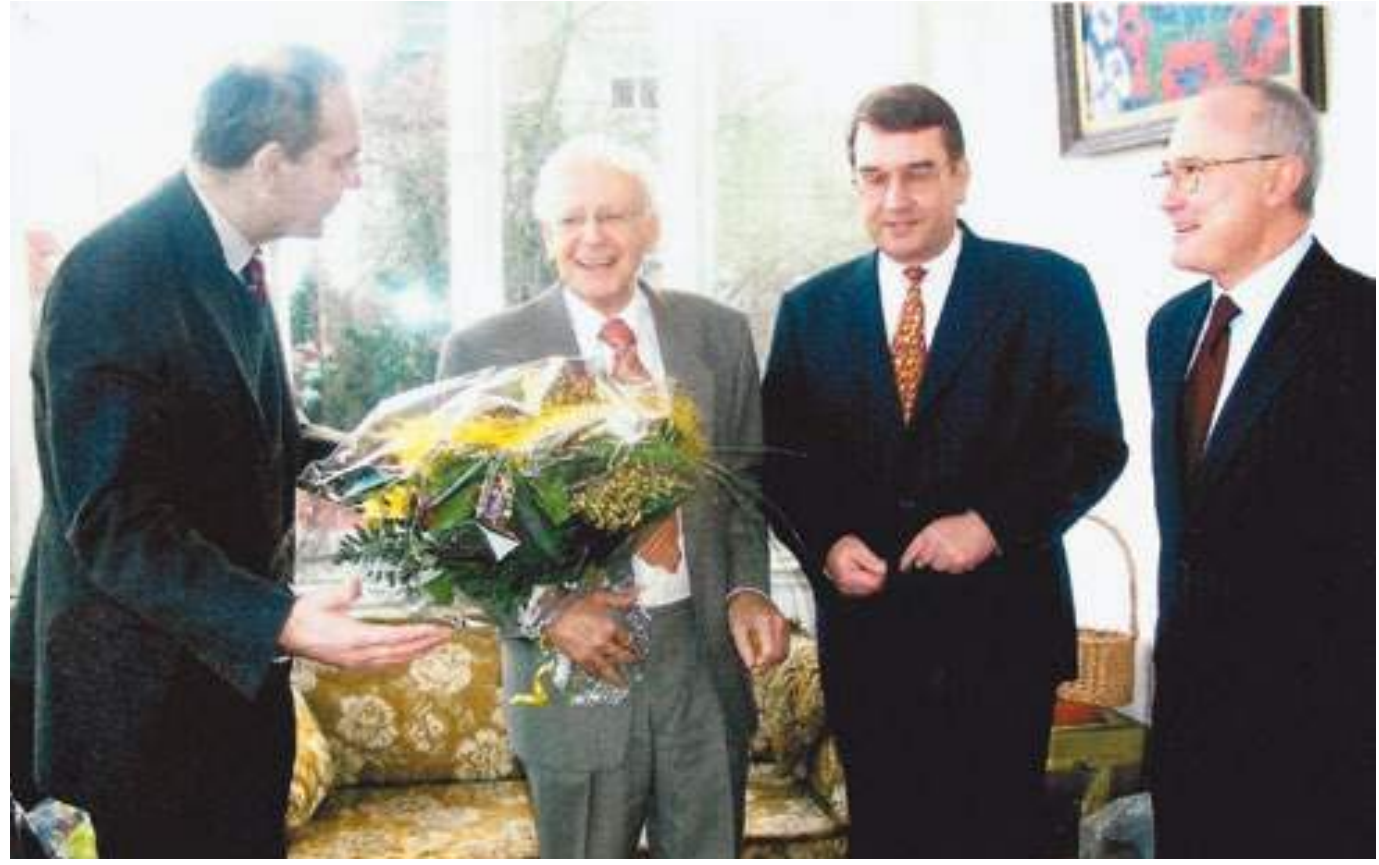

ins gesellschaftliche Leben der Stadt ein und wurde 1995 zum 1. Vorsitzenden der 1994 gegründeten Dresdner Seniorenakademie Wissenschaft und Kunst gewählt. 1998 wurde er zum Präsidenten dieser ernannt - ein Amt, das er bis 2005 ausübte. Der ehemalige Rektor der TU Dresden, Prof. Dr. Achim Mehlhorn (geb. 1939), erinnert sich: „Die Wahl Geigers zum Präsidenten der Seniorenakademie Dresden war eine gute Entscheidung. Geiger kam von außen. Er war kein ehemaliges TU-Mitglied, hatte keine akademischen Titel und trat - das hat er später im persönlichen Gespräch immer wieder betont - den pensionierten Naturwissenschaftlern und Ingenieuren mit viel Respekt und nicht ohne Herzklopfen gegenüber. Umgekehrt brachte ihm sein Charisma, seine feinsinnige Bildung, sein Engagement bei zurückhaltendem Auftreten die Sympathie und die Zustimmung der vielen Mitglieder der Seniorenakademie ein. In seinem weißen Anzug schien seine zerbrechliche Figur gleichsam über dem Auditorium zu schweben, wenn er seine Ansprachen zur Semestereröffnung hielt. Diese Reden waren immer ein besonderes Erlebnis, weil sie stets eine überraschende Botschaft enthielten, über die man lange nachdenken konnte.“3 Geiger brachte durch seine Herkunft aus dem Bildungsbürgertum und aufgrund seiner Lebenserfahrung in der DDR und der Bundesrepublik Deutschland eine Gelassenheit und Demut mit, die maßgeblich zur Versöhnung und Überwindung der Ost-
West-Konflikte innerhalb der Seniorenakademie beitrugen. 1998 gründete er die Theatergruppe der Dresdner Seniorenakademie, die bis 2002 bestand und deren Leiter er war. Mit diesem Ensemble führte er mehrere eigene Stücke auf, wobei die Laienschauspieler über die Inhalte diskutierten und Einfluss auf ihre Texte nahmen. Beispielsweise erlebte am 14. März 1999 das Schauspiel „Haus Parkweg Nr. 12“ im Haus der Kirche (Dreikönigskirche) seine Uraufführung. Die Umsetzung erfolgte mit einfachsten Mitteln - es gab kein Bühnenbild, nur einzelne Bilder wurden auf eine Leinwand projiziert. Am 29. Oktober 2001 folgte die Uraufführung des „Balladen-Theaters“ im Forum am Altmarkt (Sparkasse Dresden), und am 6. April 2002 wurde im Saal der Dreikönigskirche Geigers Schauspiel „Paula - ein Dresdner Märchen“ erstmalig gespielt.

Im Oktober 1999 erhielt Geiger von Prof. Dr. Achim Mehlhorn die erste Ehrenmedaille der TU Dresden, und am 5. Juni 2000 verlieh ihm Ministerpräsident Kurt Biedenkopf (geb. 1930) den Verdienstorden des Freistaates Sachsen. Es ist überliefert, dass Geiger diese Ehrungen auf das Tiefste bewegten. Sie gaben ihm nachträglich einen Sinn für seine Rückkehr an seine alte Wirkungsstätte Dresden.

In der Nacht vom 23. zum 24. September 2008 verstarb Erich Geiger in Cossebaude. Die 2015 erschienene Biographie würdigt sein umfangreiches Schaffen und das bewegte Leben dieses außergewöhnlichen Künstlers. 